\title{
Do the binding sites of substrates and tricyclic antidepressants overlap on the serotonin transporter? Subhodeep Sarker* and Ilka Steiner
}

\author{
Address: Institute of Pharmacology, Center for Biomolecular Medicine and Pharmacology, Medical University of Vienna, 1090 Vienna, Austria \\ Email: Subhodeep Sarker* - subhodeep.sarker@meduniwien.ac.at \\ * Corresponding author
}

from $14^{\text {th }}$ Scientific Symposium of the Austrian Pharmacological Society (APHAR)

Innsbruck, Austria. 21-22 November 2008

Published: 5 November 2008

BMC Pharmacology 2008, 8(Suppl I):A9 doi:I0.II86/I47I-22I0-8-SI-A9

This abstract is available from: http://www.biomedcentral.com/I47I-22I0/8/SI/A9

(c) 2008 Sarker and Steiner; licensee BioMed Central Ltd.

Based on the crystal structure of LeuT $_{\mathrm{Aa}^{\prime}}$ a bacterial homologue to the $\mathrm{Na}^{+} / \mathrm{Cl}^{-}$-dependent neurotransmitter transporters (SLC6 family) from Aquifex aeolicus, it was proposed that inhibitors of the serotonin and norepinephrine transporters (i.e., tricyclic antidepressants, TCAs) bind in the outer vestibule of the transporter and thus occlude access of the substrate to the substrate binding site. This model predicts that inhibitors and substrates are bound to different sites and is inconsistent with the competitive inhibition of TCA binding by substrate. We surmised that the binding sites overlapped and tested this conjecture by using carbamazepine as a ligand for the human serotonin transporter (hSERT). The underlying rationale is the fact that carbamazepine an analogue of imipramine is devoid of the aliphatic side chain. As expected, carbamazepine competes for binding of $\left[{ }^{3} \mathrm{H}\right]$ imipramine (and blocks uptake of serotonin mediated by hSERT). However, in the presence of carbamazepine, hSERT is still capable of binding substrates such as serotonin and $\mathrm{MPP}^{+}$(1-methyl-4-phenylpyridinium); this was demonstrated in experiments with $\left[{ }^{3} \mathrm{H}\right]$ imipramine as the radioligand, where the slope of the Dixon plot for serotonin and $\mathrm{MMP}^{+}$was increased upon addition of carbamazepine. In contrast, addition of carbamazepine to compounds capable of inducing transport reversal (p-chloro-amphetamine $=$ PCA, 3,4-methylenedioxymethamphetamine $=$ MDMA) invariably gave parallel shift in the Dixon plot. Based on these observations, we conclude that (i) the ring of tricyclic antidepressants does not occupy the substrate binding site, and that (ii) releas- ers induce a conformation, which precludes their simultaneous binding together with of transport inhibitors, resulting in competitive inhibition. 